\title{
La Maison de l'Amérique Latine y el Museo de Arte Contemporáneo de la Universidad de Chile: proyectos espejados para una propuesta local de la visualidad'
}

\author{
Matías Allende Contador \\ Universidad de Buenos Aires \\ mallendecontador@gmail.com
}

Recibido: 11 - junio-2018 / Aprobado: 23 - septiembre - 2018

\section{Resumen}

El Museo de Arte Contemporáneo de la Universidad de Chile, es el primer museo en coleccionar producción actual y experimental, lo que determina su nombre, en toda América Latina. Cobijado bajo la estructura universitaria, este Museo tiene la disyuntiva de su antigüedad, a su vez que presenta un programa expositivo contemporáneo. Esa discusión podría remitirse a la literatura artística de entre guerras en Francia (1918-1939), esa conexión no es azarosa, y profundizar en los planteamientos retóricos, comparados con otra institución parisina como es la Maison de l'Amérique Latine, es una tarea necesaria.

Palabras clave: Museo de Arte Contemporáneo de la Universidad de Chile, Maison de l'Amérique Latine, René Huyghe, Marcó Bontá, contemporaneidad.

1 El presente ensayo propone trabajar con los puntos de encuentro germinales entre dos instituciones que si bien importantes, y por cierto reconocidas en sus territorios específicos, carecen de un trabajo robusto respecto a su historia y las relaciones sociales, políticas y culturales, que llevaron a su creación. Es por ello que la investigación aún tiene puntos de fuga y el desarrollo de esta sigue en curso por el autor. 


\section{Abstract}

The Museum of Contemporary Art of the Universidad de Chile, is the first museum in collect current and experimental production, which determines its name throughout Latin America. Sheltered under the university structure, this Museum has the disjunctive of its antiquity, at the same time that it presents a contemporary exhibition program. This discussion could refer to the interwar artistic literature in France (1918-1939), that connection is not random, and go deeply into the rhetorical approaches, compared with another parisian institution such as the Maison de l'Amérique Latine, is a necessary task.

Keywords: Museum of Contemporary Art of the University of Chile, Maison de l'Amérique Latine, René Huyghe, Marcó Bontá, contemporaneity. 
$\mathrm{L}$ a Maison de l'Amérique Latine y el Museo de Arte Contemporáneo son proyectos que si bien ubicados en latitudes distantes del globo, confluyen en una serie de actores que, a lo largo de su devenir cultural, se encontraron repetidas veces, y particularmente su obra intelectual, tanto textual como institucional, se ha sostenido hasta el día de hoy. Los caminos paralelos pero hermanados de René Huyghe y Marco Bontá -de quienes ya nos adentraremos en sus biografías-, describen a su vez, los senderos que tomaron dos naciones, Francia y Chile, respecto a los discursos que se debían sostener en el duro contexto después de la Segunda Guerra Mundial, y en específico con la creación de estas instituciones, donde la retórica de postguerra o la Guerra Fría, se filtraría en sus propuestas museológicas y estéticas.

El ensayo se focalizará durante el período histórico francés de la Cuarta República (1946-1958), momento donde se generó en Francia una nueva infraestructura estatal en cultura, que responde a las políticas de posicionamiento del pensamiento de esa nación en el contexto occidental. Además, es un momento donde los "oficios" -denominación para ese entonces- debían alcanzar un lugar de preponderancia en su especificidad disciplinar, como lo son la museología y el cargo de conservador de museos. Para los fines de este ensayo, el historiador del arte René Huyghe (quien como veremos más adelante ejerció este cargo en uno de los museos más importantes del mundo), y el artista y fundador del Museo de Arte Contemporáneo de Chile, Marco Bontá, se envisten del cargo de conservador justamente para darle a estas infraestructuras un sentido político. De esta manera, el conservador es un canal de comunicación entre los diversos profesionales del arte, es el articulador de la estructura en general, dentro y fuera de los muros del museo.

Respecto a lo anterior, sobre la génesis del concepto de conservador, Masson Géraldine da una caracterización del personaje:

Una suma de conocimientos en historia local e historia del arte, idealmente combinado de un quehacer técnico para la investigación de las obras, es lo principalmente requerido. Un vínculo al menos tenue con el patrimonio es, además, exigido. Por eso artistas y eruditos son contratados para ser conservadores de museo $^{2}(2011,2)$.

Ese conservador primigenio al que refiere Masson, tiene las características del conservador actual en muchos lugares del continente americano, sin variar esa pericia técnica requerida, pero también en su relación orgánica y fáctica con el contexto.

2 "Une somme de connaissances en histoire locale et histoire de l'art, idéalement doublée d'un savoir-faire technique pour l'entretien des œuvres, est principalement requise. Un lien même ténu avec le patrimoine est, en outre, exigé. C'est pourquoi des artistes et des érudits sont recrutés pour être conservateurs de musée" (Traducción del autor). 
El rol profesional de conservador, nació a la vez que se masificaba y perfilaba también la disciplina museológica. ${ }^{3}$ René Huyghe, se afilió al OIM tempranamente -en 1933-, mientras trabajaba como conservador de pintura del Museo del Louvre. En esa época realizó una investigación sobre la organización de los museos a nivel europeo, de carácter inédita para el momento. Dicha investigación repercutió, como se señala en muchos estudios, ${ }^{4}$ en la creación de los museos provinciales de Francia. Me interesa proponer, particularmente y siguiendo esta premisa, cómo pudo haber repercutido esta investigación -en un sentido político y museológico más que administrativo- en los museos de América Latina. Las siguientes páginas estarán dedicadas al caso -tal vez el más evidente- del Museo de Arte Contemporáneo de la Universidad de Chile.

\section{René Huyghe}

René Huyghe (Arras, Francia, 1906- París, 1997), estudió en la Sorbonne Université cursos de filosofía y estética, y egresa finalmente de la École du Louvre para obtener la licenciatura en letras. En 1930 se vuelve el conservador adjunto del Museo del Louvre más joven -incluso hasta el día de hoy- con sólo 24 años. Posteriormente, en 1937, será el conservador en jefe de la colección de pintura. Ese mismo año, comenzó a impartir clases en la misma École du Louvre. Su fama crece a lo largo de los años, convirtiéndose en un reputado conservador, no sólo dentro de Francia, sino también en toda Europa, asistiendo a la creación de museos y otras instituciones culturales. Su práctica no sólo se restringió al Museo del Louvre, fue además curador en diversas exposiciones y dirigió revistas especializadas; sin contar numerosos libros donde participó junto a otros intelectuales.

El advenimiento de la Segunda Guerra Mundial fue un momento clave en su devenir intelectual. Su trabajo cambio absolutamente con la ocupación de Francia por el ejército nazi en 1940. Con las tropas invasoras en París, él se sumó a las tareas de protección y resguardo de las obras maestras del Museo del Louvre, para salvarlas de posibles bombardeos sobre la capital y al saqueo de los jerarcas del régimen. Junto al director del Museo en ese momento, Jacques Jaujard (1895-1967) y German Bazin (1901-1990) su adjunto en el cargo de conservador. Las obras fueron repartidas a

3 Hay que considerar el nacimiento de la Organisation International des Musées (OIM), que fue creada en 1926 por el historiador del arte francés, Henri Focillon (Dijon, 1881 - New Haven, 1943), quien hizo que esta nueva estructura pasara a formar parte del Instituto Internacional de Cooperación Intelectual (IICI), la que a su vez dependió de la Sociedad de las Naciones, estructura multilateral creada en 1920 con el fin de la Primera Guerra Mundial. Su objetivo fundamental era que, bajo esta organización, las personas relacionadas a los museos se comunicasen y pudiesen compartir diversos aspectos de la práctica institucional.

4 Estos estudios son profundizados en el texto antes citado: Géraldine, Masson. (juin 2011). Les réseaux professionnels du conservateur de musée: d'une sociabilité informelle à une organisation institutionnelle (1870 - 1940). Trabajo presentado en el Séminaire doctoral commun d'histoire de l'art et d'archéologie Paris 1/Paris 4 - 2010/2011, París, Francia. https:// hicsa.univ-paris1.fr/documents/pdf/Ecole\%20doctorale/Geraldine\%20Masson.pdf 
otras regiones de Francia. Tras esto, se une a las filas de la Résistance, lideradas por el general Charles de Gaulle (1890-1970), en contra del Estado de Vichy. Tras el fin de la Segunda Guerra Mundial fue nombrado académico de la cátedra de "Psicología de las artes plásticas" en el Colegio de Francia, en 1950.

En el plano teórico, se opuso con determinación a cualquier tesis racionalista sobre las artes. René pensaba que el arte moderno era víctima de muchas teorías sobre-elaboradas por intelectuales, que más que referir a las obras mismas, hacían gala de su potencia intelectual, lo que llevaba a lecturas en extremo funcionalistas con respecto a las obras de arte. Es por lo anterior, que eligió como su campo de estudio -dentro de la teoría del arte-, la psicología del arte, porque para él, lo aproximaba a un plano donde lo sensible era predominante dentro de la experiencia estética.

René Huyghe explicaba de la siguiente manera su metodología para la psicología del arte:

Al principio, debe impregnarse de la biografía del artista. Luego, recorrer la totalidad de su producción, puede rastrear las principales influencias estilísticas, dibujar la trayectoria de su desarrollo pictórico. Debe, igualmente, reparar en los signos que siembra su obra y considerarlos como constantes de significación particulares de ese artista $^{5}$ (Gagnon, 1987, 57).

Es decir, todo aquello que no se dice o no se manifiesta de manera evidente, aparece en la obra como testimonio de los deseos más caros del artista, pero a su vez, esa percepción velada despierta otras imágenes y representa otras sensaciones en los espectadores.

Por supuesto, la guerra como hecho primordial en la creación de los museos referidos en el presente ensayo, es una piedra angular. Varias tesis de Huyghe, propuestas desde la psicología del arte, están en uno de sus primeros libros Histoire de l'Art Contemporain. La peinture de 1935. Cuando la guerra estaba por estallar, y los nacionalismos bullían en Europa, este libro contiene una relación contextual poderosa. En dicha obra, donde trabajó como compilador de una serie de ensayos, se propone una estructura que seguía la producción de cada país o región como una forma de arte en sí misma, es decir, cada cultura o nación particular determinaba el desarrollo, en este caso específico, de su pintura. Además, y habiendo pasado casi tres décadas del cambio de siglo, para Huyghe era importante determinar un arco temporal, es decir, esta historia del arte contemporáneo únicamente abarcaría el siglo XX. Huyghe llamaba a no alterar con fórmulas el complejo tiempo en el que se estaba viviendo. Cualquier tipo de esquematismo cerraría la producción visual a una serie de nombres, las cuales no significarían pregunta alguna para el futuro.

5 "Dans un premier temps, il doit simprégner de la biographie de l'artiste. Ensuite, en parcourant la totalité de sa production, il peut retracer les principales influences stylistiques, refaire la courbe de sa progression picturale. Il doit, également, repérer les signes qui parsèment son œuvre et les considérer comme des constantes de signification particulières à cet artiste”(T.del A.). 
Sin querer ser nacionalista -mucho menos juzgar una propuesta compilatoria novedosa y germinal dentro de la literatura artística de Occidente-, los "tiempos de orgullo" como señalaba Huyghe en esta obra, cobraron fuerza no únicamente en forma plástica, sino también social y militarmente, deviniendo la guerra. Algo interesante, es que Histoire de l'Arte Contemporain fue uno de los primeros libros en referir una visualidad localizada en América Latina. Dentro del libro se dedica un pequeño apartado, escrito por el mismo Huyghe, a la argentina Norah Borges, y a los uruguayos Rafael Barradas, Pedro Figari y Joaquín Torres-García, además del chileno Ortiz de Zárate ${ }^{6}$. Sin embargo, para Huyghe (1935) son solamente los mexicanos con su muralismo, quienes lograron conseguir lo que él denominó una "expresión única".

Histoire de l'Arte Contemporain contó también con una colaboración importante de Germain Bazin, un prefacio del político y escritor Jean Mistler, y una introducción de Henri Focillon. Dentro de estas primeras páginas de Focillon, el investigador se refiere a las intensas diatribas respecto al arte moderno en lo que se llevaba del siglo XX, y propone que el interés principal no son los artistas, sino más bien, el cómo las obras de arte podían ser reflejo de la personalidad de estos y al mismo tiempo ser un medio de expresión de su contexto, saliendo de la simple apreciación subjetiva y enarbolando un conocimiento positivo, cómo señalaba Mistler, del terreno en disputa que es la apreciación estética $(1935,2)$.

Siguiendo la idea anterior, se señalaba que:

“¿Qué es esa curiosa noción de modernidad que ha trabajado las conciencias en Occidente desde más de un siglo y que alcanzó, quizás, hace poco toda su intensidad, de lo contrario una suerte de instinto histórico confuso (a veces endurecido), un sentido de las necesidades del momento, una impaciencia de las formas usadas, que intentan precipitadamente novedad? El arte busca así descubrir, en el crear, su actualidad"7 (Focillon, 1935, 5).

Es así como las características formales son relegadas. Para ellos la idea de vivir el momento y expresar el contexto inmediato se vuelve la necesidad primaria de la obra de arte, y esto, a su vez, se vuelve el problema determinante del futuro. Así, la escritura de arte actual debe sentar las bases de la crítica de arte futura.

René Huyghe en esta y otras obras señala que la crítica de arte futura tiene la necesidad de conocer los instintos, las costumbres y los acontecimientos que tienen o realizan los artistas para producir obras, pero, sobre el mismo sentido, es importante

6 Sin dejar claro si se refiere a Julio o Manuel, instalados largamente en París.

7 "Qu'est- ce donc, cette curieuse notion de modernité qui travaille les consciences en Occident depuis plus d'un siècle, et qui atteignait, peut-être naguère toute son intensité, sinon une sorte d'instinct historique confus (et parfois durci), un sens des besoins du moment, une impatience des formes usées, qui en tente précipitamment de nouvelle? L'art cherche ainsi à découvrir, à créer son actualitê" (T. del A.) 
conocer el pasado en tanto cimientos de propuestas respecto del tiempo actual, y no necesariamente hacia la obra. Sin fórmulas, sino más bien marcos, se definirían las obras de la siguiente forma: “¿Pero entonces no habría reglas, definiciones del arte? No, no hay que normar, no hay definiciones, sólo hay disciplinas sucesivas, diferentes, que a veces se reproducen, puesto que la variedad no es infinita" (Huyghe, $1949,6)$. Está máxima es una afrenta a la difundida historia de los estilos dentro de la historia del arte, negando cualquier posibilidad de que un grupo, escena o generación, defina o dignifique el gusto de un conjunto humano, sobre todo considerando las posibilidades de los mismos.

Esta crítica a la "clasificación" de los artistas, se la atribuye Huyghe, como ya señalábamos a la teorización de la producción creativa: “(...) en nuestro tiempo, se sirven de ideas en lugar de servirles; las usan para justificar su causa, sus pasiones, sus sectarismos; el pensamiento es en este punto reducido de su condición de diseño, en esto, instrumento de objetividad, no tiene más sentido para muchos entre nosotros"9 (ibíd.). Huyghe presenta esta necesidad ante una fuerte conciencia de las diversas tendencias que se cruzan en el momento de la primera mitad del siglo XX. En muchos casos, estos movimientos no permitieron presentar el crisol de producciones artísticas de los contextos específicos.

René Huyghe es uno de los iniciadores de una nueva historia del arte, que se define por girar el lugar de la práctica disciplinar, desde la escritura y su compilación literaria, a la definición y consolidación del rol activo del espectador, ubicando la historia del arte en un lugar donde predomina el acto de mirar por sobre el escribir. La importancia de la aprehensión inmediata del acontecimiento estético. La velocidad de los tiempos actuales demarca el acto de mirar en un summum total de experiencias traducibles e identificables con el espectador.

Esta propuesta, podría redondearse en la idea de generar una metodología para la historia del arte per séy sin interrogantes del pasado, que los historiadores, como intérpretes de las obras y la experiencia de los espectadores, sean sujetos de su propia historia. Lo anterior es algo imposible para las tesis positivistas del momento, y también, para quienes - dentro de las tesis formalistas, por ejemplo- abrazaban el materialismo dialéctico, como Arnold Hausser (1892-1978), tesis ampliamente difundidas en la Europa de principios de siglo XX. Para Huyghe, estas eran el resultado de las desgracias sociales y económicas del presente y no respondían necesariamente a los pensamientos de los artistas. Entonces, para René Huyghe “(...) la historia del arte contaba con la posibilidad de la experiencia directa, una percepción ajena a la disciplina de la historia. De este modo, demostraba la li-

8 "Mais alors n'y aurait-il pas de règles, de définitions de l'art? Non, il n'y a pas de régler, pas de définitions, il n'y a que de disciplines successives, différents, que parfois se reproduisent, car la variété n'en est pas infinie” (T. del A.)

9 “(...) en notre temps, se servent des idées au lieu de les servir ; ils s'en servent pour justifier leur causer, leurs passions, leurs sectarismes; la pensée est à ce point réduite de sa condition de dessin à celle d'instrument que l'objectivité n'a plus de sens pour nombre d'entre nous" (T. del A.) 
mitación de los discursos históricos positivistas que daban más importancia a los documentos, a los contratos, a las firmas y a las fechas que a los objetos artísticos. $\mathrm{Al}$ respecto, Huyghe explica: "«el documento escrito primordial en historia, no es más, no debe ser aquí más que el material infinitamente útil, es cierto, pero anexo o preliminar»" (Barreiro-López, 2015, 19).

Huyghe toma de Henri Focillon -antes mencionado- la idea de "tiempo histórico", donde la obra de arte se dirime en la disputa de ser un objeto atemporal y también ser parte del devenir del tiempo, puesto que ha sido producido y su forma responde a una serie de acontecimientos que recoge y desarrolla desde un momento específico. Sin extendernos en dicho concepto, es importante mencionarlo, ya que es allí donde dos tesis de Huyghe podrían sustentarse, una: la importancia singular del artista, en tanto sujeto consciente del contexto social, y otra: la preservación de las obras de arte, cómo testigos (o testimonios) de un tiempo específico de la historia. Huyghe fue el gran promotor de un taller de restauración en el Museo del Louvre, a su llegada como conservador. Desde ahí defendió el lugar central que tiene la obra de arte en el museo, sobre cualquier otra área, es decir, su conservación era primordial. Además, dentro de la práctica museológica, particularmente el comisariado de exposiciones, derivaba en una forma de interpretación histórica con los objetos, siendo sus trabajos en ese sentido, una de las aristas más estudiadas de su obra.

Esta visualización no escrita, la narración histórica completada por el espectador, pero hilvanada por el comisario, es algo que sustenta su tesis respecto al cambio de paradigma civilizatorio, desde la escritura a la mirada. Una mirada que forja, sobre cualquier otra cosa, una identidad cohesionada en un espacio específico: el museo; y que, a mediados del siglo XX, era sin lugar a dudas el terreno en disputa.

\section{Museo de Arte Contemporáneo de la Universidad de Chile}

El Museo de Arte Contemporáneo de la Universidad de Chile se inauguró el 15 de agosto de 1947, siendo el primer museo en denominarse así, es decir de arte actual, en toda América Latina. Las condiciones para su creación se generaron dentro de un plan mayor de resonancia territorial de la Universidad, por dos factores determinantes, uno: demostrar fácticamente la condición "nacional" de la Universidad de Chile; y dos: generar una visibilidad de la institución en el plano local y regional, en relación a sus cien años de historia cumplidos para ese decenio. Para lograr dicho plan se ocupó la extensión cultural como gran arma en el posicionamiento de la Universidad, la cual no se restringió únicamente a las artes, sino a los saberes en general, generando instancias pedagógicas que más tarde devendrían en sedes regionales de la misma Universidad.

En 1949, se individualizaron las instituciones que llevarían a cargo la labor de la difusión universitaria en la sociedad, especialmente a través de los servicios o departamentos de extensión, en cuya ausencia se crearían Comisiones especializadas con ese fin. Por otro lado, los entes 
que llevarían a cabo la misión de la extensión cultural eran el Departamento de Difusión Cultural, el Departamento de Escuelas de Temporada y Cursos de Extensión Universitaria, el Departamento de Teatro Nacional, el Departamento de Bibliotecas y el Departamento de Enseñanza Popular Valentín Letelier. También fueron considerados en este grupo el Departamento de Publicaciones, el Instituto de Extensión Musical y el Instituto de Extensión de Artes Plásticas (Berríos y Cancino, 2018, 181).

Cada institución antes mencionada por Berríos y Cancino, fue creada antes del final de la década de 1940, y sin embargo todas, dentro del rectorado de Juvenal Hernández Jaque (Chillán, 1899 - Santiago, 1979), entre 1933 y 1953. Hernández es considerado el continuador por excelencia del proyecto del fundador de la Universidad, el intelectual venezolano Andrés Bello, quien en 1842 da apertura a una institución que tiene como eje, dirigir todas las áreas del saber nacional. Hernández profundiza dicho proceso en la extensión nacional de la Universidad, pero sobre todo, en la creación de infraestructuras que pudiesen trabajar exclusivamente en dichas tareas.

La gestión de Juvenal Hernández, responde a una política generada en Chile desde finales de la década de 1930, de invertir e incentivar la industria nacional. Es así como las universidades, particularmente la Universidad de Chile, ya no fueron más instancias de producción académica o formativa, sino que comenzaron a enseñar disciplinas "aplicadas" a las necesidades propias de un Estado desarrollista. ${ }^{10}$

El proceso de consolidación de las artes aplicadas, sin desmedro, pero sí a desazón de las bellas artes, fue incentivado por una serie de artistas que, en 1928 y tras un polémico Salón Nacional, fueron becados por el Estado para realizar cursos de perfeccionamiento en Europa por uno o dos años. El producto de dicho proceso fue una serie de jóvenes artistas formados en grabado, textil, cerámica, forja o platería (en París, Berlín, Madrid, entre otras), y que volvieron con el deseo de cambiar las bases institucionales donde ellos se habían formado originalmente. Su regreso como docentes fue evitado por los profesores ya entronizados en las cátedras, y su única opción fue la defensa y consolidación de la Escuela de Artes Aplicadas. Dentro de los artistas jóvenes formados en artes "útiles" y que, desde el principio de la década de 1930, dedicaron su fuerza laboral a dicha Escuela, estuvo Marco Bontá (Santiago de Chile, 1899-1974).

Bontá, junto a los becarios partió de Chile en 1928, después de haberse formado en la Escuela de Bellas Artes. Si bien estudió en varias capitales europeas, fue en París donde se perfeccionó en grabado, disciplina que desarrollaría toda su vida, a la par de la pintura. A su regreso en 1930 fue un activo promotor del cambio de docentes en Bellas Artes, sin embargo no lo logró, y en 1931 fundó y asumió la cátedra de

10 Dicho proceso, si bien en la Escuela de Bellas Artes fue anterior a 1930 con las cátedras de "Artes decorativas" y "Artes aplicadas", no es, hasta la especificidad alcanzada con las materias impartidas en la Escuela de Artes Aplicadas (como cantería o técnicas útiles), y la radical distancia que tomó dicha Escuela de su par en Bellas Artes. 
grabado en la Escuela de Artes Aplicadas y en la Universidad de Chile. Tras esto, en 1938 fue contratado junto a una serie de otros artistas e intelectuales chilenos en las "misiones pedagógicas chilenas", por el gobierno venezolano, iniciativa que se extendió hasta 1946 (e inclusive un poco más), para la reformulación de la Escuela de Bellas Artes, Artes Aplicadas y el Instituto Pedagógico. Dichas misiones fueron lideradas por el escritor venezolano Mariano Picón-Salas (Mérida, 1901- Caracas, 1965), quién se formó en Chile, tras huir junto a su familia de la dictadura de Juan Vicente Gómez. Picón-Salas, quien fue profesor de historia del arte en la Escuela de Bellas Artes, llamó a sus ex colegas para involucrarse en la reformulación de la educación venezolana, por la alta estima que tenía a la institucionalidad chilena.

Bontá a su regreso en 1945, fundó el Instituto de Extensión de Artes Plásticas (IEAP), que debía difundir la creación visual a nivel nacional, y a su vez, apostar por su internacionalización. ${ }^{11}$ Se cobijó al alero del IEAP el MAC, que fue fundado por su mismo director Marco Bontá, quien dejó la administración del Instituto en 1949 para dedicarse exclusivamente el Museo. Dentro de las directrices que planteó Bontá para el MAC, estaba ser un museo dedicado al "(...) último medio siglo, sin discriminación de estilos, con el fin de que represente en la forma más clara y completa un panorama de la creación artística viva del país" (Bontá, 1957, 16). Se generó, por lo tanto, un museo que difundía la producción de los creadores vivos, sin especificidad en cuanto a campo disciplinar, bellas artes o artes aplicadas, las cuales representaron un baluarte para la identidad nacional.

Es así como se tiene que comprender el MAC bifurcado en dos caminos, la experimentación por un lado, y las artes abocadas a la sociedad deslindadas de los circuitos académicos por otro, aun cuando estaba -y está- en un organismo de estas características. Contradictorio por donde se le mire, pero al mismo tiempo trata de conformar una retórica propia de las distancias que se intentaron construir desde finales de la segunda guerra mundial, con los desvencijados estados europeos, y en concordancia con los fundamentos narrativos de la raza americana (Allende, 2018, 96).

Recordemos que el MAC colecciona y exhibe el mismo arco temporal propuesto por René Huyghe en Histoire de l'Art Contemporain, sin antecedentes institucionales previos, reforzando la noción de darle un carácter particular al siglo XX. Entonces, es interesante que la creación del Museo de Arte Contemporáneo, cómo señalan en su libro Un tiempo sin fisura Berríos y Cancino, se estableció como un hito fundamental por el robustecimiento de una estructura estatal que consolidaba el discurso nacional-desarrollista que se expandida por toda América Latina, y que para mediados de la década de 1940, en Chile, se presentó con contundencia.

11 Sin embargo, el primer antecedente de un organismo como este, es su predecesor dentro de la misma Universidad de Chile, el Departamento de Extensión Cultural, que desde 1930 fue creado y dirigido por Tomás Lago (Chillán, 1903Santiago de Chile, 1975). Lago, quien también fundó en 1942 (mismo año del Centenario de la Universidad), el Museo de Arte Popular Americano (MAPA), que con la creación del IEAP, fue asimilado por esta estructura mayor en tamaño. 
Las labores del IEAP no sólo consistían en aglutinar las instituciones de difusión de la visualidad, sino también la organización de exposiciones en otros organismos con características similares, como es el caso de la exposición del pintor uruguayo Pedro Figari en el Museo Nacional de Bellas Artes en Santiago. El Instituto logró ser una estructura modernizadora del quehacer museal y exhibitivo, permitiendo la circulación nacional e internacional, con contactos tanto dentro como fuera del país. Sin embargo, lo anterior se sustenta a su vez por una débil infraestructura presupuestaria dentro de la Universidad, que llevaba al Museo a depender de exposiciones foráneas, para llenar la programación de las instituciones:

No obstante, el clima de colaboración entre instancias estatales y privadas para la tarea de la difusión de la cultura no subsanó necesariamente los infortunios presupuestarios que padecían el Instituto y Facultad. Por ello, buena parte de las gestiones de los directores del IEAP, en especial la de Marco Bontá, se abocaron a la obtención de fondos o alianzas que permitieron llevar a cabo la misión del organismo a cabalidad. Este tipo de apoyo en la liberación de impuestos de aduana, en el traslado de exposiciones internacionales (...) (Berríos y Cancino, 2018, 193-194).

En esta relación de estructuras económicas y puesta en valor de una visualidad nacional, germinó el Museo de Arte Contemporáneo, siendo ese modelo el que se consolidaría con el tiempo. Entre exposiciones de artistas locales jóvenes y artistas consolidados del exterior. Sin embargo, cabe preguntarse ¿a quiénes respondían estas programaciones en términos de la visualidad como un campo de disputa durante la Guerra Fría?

\section{Maison de l'Amérique Latine}

La Maison de l'Amérique Latine nació en la histórica reunión del Quai d'Orsay, convocada por Etienne Dennery (director de asuntos sobre América del Ministerio de Relaciones Extranjeras y antiguo director de Servicios de Prensa de la Francia Libre). A ella llegaron sobre todo personalidades de La Résistance (gaullistas incorporados al nuevo gobierno). Es importante detenernos en la relación entre la Francia Libre (La Résistance) y los países latinoamericanos durante la guerra, particularmente porque la mayoría de los aportes económicos para el ejército de Charles de Gaulle provinieron de nuestra región (de hecho, de los 400 comités de ayuda a La Résistance, 300 eran latinoamericanos). ${ }^{12}$ Hay dos características esenciales que

12 Esta ayuda solicitada por De Gaulle durante la guerra, si bien tiene esa repercusión evidente en la creación de la Maisonde l'Amérique Latine en París, el gesto más importante que tuvo el general y presidente francés, fueron las series de misiones diplomáticas en nuestra región, iniciadas en 1961. Las personas encargadas durante la guerra en solicitar, afianzar y reconocer públicamente estos aportes las naciones americanas, fueron: Louis Pasteur Vallery-Parot, presidente del comité médico de La Résistance, y Robert de Billy, abogado, aviador, una persona inmersa fuertemente en el mundo cultural latinoamericano, además fue en uno de sus castillos donde se ocultaron obras del Museo del Louvre (durante la operación de Jaques Jaujard y René Huyghe). Ambos posteriormente fueron fundadores de la Maison del'Amérique Latine. 
relacionan a los fundadores de la Maison. En primer lugar, algún tipo de vínculo (cultural, político o afectivo) con nuestro continente y, en segundo lugar, una fuerte admiración al general Charles de Gaulle. ${ }^{13}$

La fundación de la Maison de l'Amérique Latine es una respuesta a la necesidad de Europa, y particularmente de Francia, de resistir y consolidar relaciones con países que tenían grandes capitales humanos y naturales. Ante el sabido deterioro de ese continente empobrecido tras la guerra, y donde el único vencedor parecía ser los Estados Unidos (quienes generaban una poderosa influencia en América Latina); La Maison se genera como una contra-respuesta europea de esta influencia. Por esto, para la Maison, "(...) fue necesario recibir a sus dirigentes y sus élites en las mejores condiciones, demostrándose nuestro afecto a sus países" ${ }^{14}$ (Rouquié, 2016, 6). Por lo tanto, la Maison es ante todo una misión diplomática.

La Maison de l'Amérique Latine abrió con una exposición de arte latinoamericano titulada Ars Americana, desarrollada entre el 18 de octubre y el 5 de noviembre 1946. La muestra estuvo centrada en tres países: Chile, Argentina y Uruguay, con el patronazgo de cada una de las embajadas en París. Antecedentes en París para una exposición de arte latinoamericano, previos a la existencia de la Maison, no son muchos, particularmente porque en Francia no había un interés puntual por lo producido en nuestra región y, por otro lado, son los artistas latinoamericanos, en muchos casos de clases acomodadas, quienes viajaban a perfeccionarse e insertarse en el mundo parisino, asimilando diversos procedimientos de obra, como dictaba el canon de la época. ${ }^{15}$

Para la exposición, cada país conformó una comisión para seleccionar las muestras de visualidad más específicas de cada nación. ${ }^{16}$ René Huyghe escribió el texto principal y se envistió a sí mismo como el comisario de la muestra, siendo muy probablemente, la persona que más contactos tenía con la visualidad latinoamericana. El texto es, por cierto, una profundización épica a la relación del mundo latino entre sí, y el compañerismo que se debía trabajar en tiempos de guerra. Inicia con un análisis de la conformación cultural de América del Sur, señalando que nuestro continente se compone de dos partes, el nor-pacífico, como él dijo "marcado por las

13 La lista completa de fundadores de la Maison de l'Amérique Latine, fueron: Étienne Dennery (1903- 1979), Paul Rivet (1876- 1958), Louis Pasteur Vallery- Radot (1886- 1970), Luiz Martins de Souza Dantas (1876- 1954), Jean Casson (1897- 1986), Robert de Billy (1894-1991).

14 “(...) Il faut accueillir ses dirigeants et ses élites dans les meilleures conditions, leur montrer notre attachement à leur pays" (T. del A.)

15 Dentro de las exposiciones que se pueden destacar está la Exposition d'art Américain-Latin en marzo de 1924, en el Museo Galliera; y la Exposition d'art Argentin en 1926, en el Museo del Jeu de Paume.

16 Para el comité argentino participó Rodolfo Alcorta, Alfredo González Garaño, Julio E. Payró; en el uruguayo Giselda Zani, Clotilde de Podestá, Julien Coffinet; y finalmente en el chileno Marta Brunet, Marco Bontá y Tomás Lago. La exposición fue organizada por Gisèle Freund y René Hubert, con un comité compuesto por Louis Joxe, Georges Salles, René Huyghe, Germain Bazin y Raymond Ronze; finalmente cada país tuvo un comité de honor compuesto por tres intelectuales franceses de diversas áreas. 
civilizaciones antiguas", y por el otro lado, la parte sur-atlántica (desde Brasil hasta inclusive Chile), sería la nueva civilización. Posteriormente, se sumerge en el devenir de la plástica en el continente señalando que: "Los intentos de un arte autónomo, "colonial», análogo a ese "primitivo» de América del Norte, carecen de futuro. Resultaron en un hiper-provincialismo que, cortado de sus fuentes europeas, recayó en una sabrosa torpeza" ${ }^{17}$ (Huyghe, 1946, 12). Como vemos, sin despreciar absolutamente la producción anterior a las repúblicas, si las caracteriza, casi de anecdóticas, logrando al parecer, para Huyghe, una localidad extremada.

La retórica propuesta por Huyghe, deviene en el texto en la consagración de la cultura latina y específicamente en la cultura latinoamericana, siendo un vergel de posibilidades creativas. Declaró en el texto que cualquier pretensión colonialista de parte de Europa con este continente, no es entender su potencia particular:

¡Qué problemas se instalan y aún están pocos definidos! El de los europeos por continuar con cuatro siglos de retraso el espíritu de los españoles de antańo y concebir América del Sur como un simple anexo a su cultura. En realidad, ese continente es la intersección más prodigiosa, donde se encuentran todas las razas del mundo. El genio latino, ha sido felizmente marcado hasta el alma, su fe emocionante en el humanismo y en la vida espiritual le proporcionan los medios para realizar esta basta síntesis, en la cual lleva en sí los elementos aún caóticos y sofocantes. Su tarea es grande, ruda y difícil. El triunfo sólo podrá ser de consecuencias inmensas. Para alcanzarlo, se necesita que la América del Sur no sucumba a las influencias externas; la hora de su madurez ha llevado; se precisa aún que la América del Sur armonice los elementos divergentes de su "complejidad". Tiene ante sí, en ella, los elementos de un arte, y más fuertemente: de una civilización ${ }^{18}$ (Huyghe, 1946, 18).

La égloga sobre América Latina no es nueva, sin embargo, son las naciones americanas quienes produjeron esta retórica edénica, incluso babilónica en su multiculturalidad y diversidad racial, antes que los europeos. Así como René Huyghe escribe un libro inaugural de la historia del arte contemporáneo, nos atreveríamos a señalar, que es uno de los primeros en darle la "estatura histórica" a América Latina viniendo de Europa. Siendo para él un territorio otro, pero absolutamente cohesionado culturalmente, en eso llamado la "latinidad".

El catálogo tiene un poema de Pablo Neruda titulado Arte popular, y un texto de Tomás Lago sobre el Arte popular chileno. Además, la cuentista, poeta y diplomática

17 "Les essais d'un art autonome, "colonial", analogue à celui des "primitifs" de l'Amérique du Nord, sont sans lendemain. Ils aboutissent à un hyper-provincialisme qui, coupé de ses sources européennes, retombe à une savoureuse gaucherie” (T. del A.)

18 "Que de problèmes posés et encore indistincts! Il est des Européens pour continuer avec quatre siècle de retard l'état d'esprit des Espagnols de jadis et pour concevoir l'Amérique du Sud comme une simple annexe de leur culture. En réalité, ce continent est le plus prodigieux carrefour, où se rencontrent toute les races du monde. Le génie latin, dont il a été heureusement marqué jusqu'à l'âme, sa foi émouvante dans l'humanisme et la vie spirituelle lui apportent les moyens de réaliser cette vaste synthèse dont il porte en lui les éléments encore cahotiques (sic) et étouffants. Sa tâche est grande, rude et difficile. La réussite ne pourrait être qu'immense de conséquences. Pour y atteindre, il faut que l'Amérique du Sud ne succombe pas aux influences extérieures; l'heure de sa maturité est venue; il faut encore que l'Amérique du Sud harmonise les éléments divergents de son "complexe ". Elle a devant elle, en elle, les éléments d'un art, et davantage : d'une civilization" (T. del A.) 
uruguaya Giselda Zani, en el mismo catálogo recoge las pretensiones expresadas por Huyghe y, es más, se refiere al rol de los artistas, específicamente los chilenos, uruguayos y argentinos, en esta tarea:

Si el destino de América Latina es, como nosotros creemos, este de ser la heredera y la colaboradora de la cultura occidental, con todo eso que esta trae consigo de universalidad, de humanismo, de rechazo de nacionalismos intolerantes, de búsqueda de los valores absolutos del espíritu por encima de toda contingencia, y si las artes plásticas tienen alguna parte de esta enorme tarea, Uruguay, Argentina y Chile, quienes han dado a luz a tantos artistas que viven bajo el signo de este fin, se puede decir que ellos han, así, culminado una gran parte del rol que les pertenece ${ }^{19}$ (Zani, 1946, 19).

La tarea, por cierto, ambiciosa, es resarcir el humanismo perdido. El siglo de las luces que tanto enorgullecía a Francia, y que convirtió a su capital París, en el faro de Occidente. Sin profundizar en cuán efectivo fue la tarea de la Maison, mucho menos determinar un éxito o no en ello, sí podríamos señalar, que en la retórica de René Huyghe, desde la creación de Histoire de l'art Contemporain hasta su texto en Ars Americana, hay una defensa de la producción visual como mensaje epocal, que construye una identidad colectiva fuera de cualquier populismo político o retórica económica.

Este Proyecto, la Maison, se enmarcó en una serie de iniciativas donde Francia intentó recuperar o fortalecer su actividad cultural, y robustecer el carácter francófilo en el mundo latino. No es azar que desde 1946, y sobre todo en 1947, se abrieron las sedes por países del Institute de France en América del Sur. ${ }^{20}$

\section{Bontá y una contemporaneidad posible}

Ahora bien, el presente ensayo se sustenta a partir de varias situaciones que aun cuando parecen anecdóticas, se cruzan en varios momentos históricos, y ciertas propuestas que se repiten y cambian de estructura, de la escritural a la institucional, en Santiago o París. Es interesante el contexto político, marcado por dictaduras, tanto en Europa como en Venezuela, y que al finalizar llevaron a la creación de nuevas organizaciones gubernamentales en cultura.

En 1935, se publicó Histoire de l'Art Contemporain. La Peinture dirigido por René Huyghe, un par de años antes de estallar la guerra que cambió el panorama cultural del mundo completo durante el siglo XX. Sin ser azaroso, Marco Bontá y

19 "Si le destinée de l'Amérique Latine est, comme nous le croyons, celle d'être l'héritière et la collaboratrice de la culture occidentale, avec tout ce que celle-ci comporte d'universalité, d'humanisme, de refus de nationalismes étroits, de recherche des valeurs absolues de l'esprit au-dessus de toute contingence, et si les arts plastiques ont quelque part dans cette énorme tache, l'Uruguay, l'Argentine et le Chili, qui ont donné naissance a tant d'artistes qui vivent sous le signe de cette finalité, peuvent se dire quils ont, ainsi, accompli une grande part du role [sic.] qui leur revient" (T. de A.)

20 Además, esta infraestructura gubernamental francesa, superó con creces el mero interés cultural, creando en el 1946 la Cámara de Comercio Francia-América Latina y el grupo Parlamentario de amistad Francia-América Latina, en 1947. Sin embargo, todas estas iniciativas no fueron suficientes para disputar el lugar de preponderancia política y económica a los Estados Unidos; una carrera que, por cierto, hacia la década de 1960 Francia deja casi por completo. 
René Huyghe, colaboraron muchas veces a lo largo de su vida, y ambos trabajaron - por ejemplo- en el desarrollo de los institutos nacionales, el francés y el chileno, la Académie Française en el caso de Huyghe (por ser escritor) y la Academia de Bellas Artes, Marco Bontá (al ser artista).

Sin embargo, es la Segunda Guerra Mundial, el hecho que se repite, constantemente tanto en los textos de Huyghe, como en las condiciones de transferencia y traslado cultural, entre América Latina y Francia.

Ahora, pareciera que el problema que arrastra el Museo de Arte Contemporáneo, en términos epistemológicos más que museológicos, es justamente el que Giorgio Agamben señala como pregunta inicial en su ensayo ¿Qué es lo contemporáneo? (Agamben, 2012, 1). El llamado a Friedrich Nietzche de Agamben es para que consideremos en primera instancia cuál es el lugar dónde se posicionan los sujetos respecto a su propio relato, es decir, en relación al devenir histórico que se presenta en el momento actual de la cultura. Esa cultura, es la que hay que hacer entrar en consideración. Esa lectura a la "consideración" nietzcheana de Agamben podría leerse como el desplazamiento del sujeto en el arco histórico, aun cuando no hay espacio en ese tránsito que supone la vida dentro de la narración general de la historia, este sujeto se moviliza igual; podríamos pensarlo como una salida del canon.

Agamben ve un coeficiente de oportunidad en la "oscuridad" del momento histórico que pudo cada uno vivir, es decir, el sujeto contemporáneo trabaja fuera de las zonas de confort, y más bien remueve las aguas para alcanzar el espeso barro que carga con los acontecimientos del pasado. Esto es interesante, porque según la narración que ha adquirido las carillas acá presentadas, la segunda Guerra Mundial presentó -nunca desde una perspectiva positiva, ni mucho menos- con su fin, la condición de superación social y cultural, que el general De Gaulle, René Huyghe y La Résistance francesa en general, tomaron para proyectar una nueva Francia. El impacto de la misma, en una reverberación epocal llegó hasta la austral Santiago de Chile, y determinó el impulso histórico para la creación de un Museo de Arte Contemporáneo.

Lo contemporáneo, en el texto de Agamben, es también la capacidad que este sujeto pueda tener de desplazarse en la historia -como ya decíamos-, y, sin embargo, desplazar también otras historias, otros tiempos, es así como los pone en relación, diálogo y comunicación, como una necesidad de escarbar esas zonas oscuras; que a su vez, definen probablemente, la identidad y su carga socialmente determinante. Esto devendría, posiblemente, en el problema de la relación de la tradición con el acontecimiento histórico y un "coeficiente de oscuridad".

Dado que el presente no es otra cosa más que lo no-vivido de todo lo vivido y que impide el acceso al presente es justamente la masa de lo que, por alguna razón (su carácter traumático, su demasiada cercanía), no logramos vivir en él. El cuidado puesto a esto no-vivido es la vida del contemporáneo. Y ser contemporáneos significa, en este sentido, regresar a un presente en el que nunca hemos estado (Agamben, 2012, 4). 
Entonces, la contemporaneidad para Agamben, es una presencia del sujeto en la objetualización de un acontecimiento, es determinar que en esa novedad dentro del arco del tiempo existe algo indescifrable. Lo moderno, entonces, en tanto que configura un lenguaje nuevo, también en su inscripción en su ser estar en el tiempo, lo deja obsoleto. "La contemporaneidad se inscribe en el presente y lo marca, ante todo, como arcaico, sólo quien percibe en lo más moderno y reciente los indicios y las marcas de lo arcaico puede ser contemporáneo" (Agamben, 2012, 3). En ese sentido, en esa frase indudablemente poética y enrevesada de Agamben, lo que podemos comprender, es una latencia de lo arcaico en lo moderno, o sólo lo realmente moderno, es decir, contemporáneo, puede descifrarse desde allí. Entonces, una pregunta medular en el texto, que lo cruza, pero no se aterriza hasta ahora, es ¿cuándo el arte moderno pasa a ser contemporáneo? Una lectura de superación que actualmente, en un relato de grandes marcas, fija en la escritura -pero no en la visualidad-, un antes y un después.

Entonces, lo contemporáneo, en tanto provoca la obsolescencia del tiempo, en su escritura o institucionalización, detiene lo moderno, pero lo detiene en un momento determinado, ${ }^{21}$ por lo tanto, hay una cohabitación entre lo contemporáneo y lo moderno, o para decirlo más claramente, entre el arte moderno y el contemporáneo no habría ninguna diferencia. Entre los dispositivos creados por René Huyghe, su teoría sobre la historia del arte contemporáneo, y Marco Bontá, con la creación de un Museo de Arte Contemporáneo, en el país más austral del mundo, que seguía recibiendo los influjos postimpresionistas para la década de 1940; la obra de arte contemporánea no supera la modernidad, sino que graba las condiciones de posibilidad de dichas obras.

No significa que en esa apertura epistemológica se encuentre, aún hoy, el arte contemporáneo. La superación de arte moderno no ha sido porque el arte contemporáneo ha derivado en "post" o "extremo", como se suele seńalar en la historia del arte local hegemónica. Por el contrario, el arte moderno ha envejecido en formas, estrategias y discursos que sirven para marcos escuetos, y que si bien se relacionan con su momento, no son estrictamente -estas obras- dispositivos que comparezcan con el contexto, inclusive, no son conformadores de identidad. Huyghe se refiere en 1949, a los artistas que concomitan con las obras modernas, señalando:

De otra manera, pronto el arte moderno tendrá que soportar otro examen aún más severo y donde se reflejarán las parcialidades nacidas de las condiciones nuevas: el futuro se aproxima, habrá cambiado, padecerá de otras fatigas, de otros deseos, y reprochará en el arte de hoy de ser solidario a ese pasado que, sin embargo, ha creído destruir ${ }^{22}$ (Huyghe, 1949, 116).

21 Las coincidencias posibles entre el concepto de "tiempo histórico" de Henri Focillon y "contemporaneidad" en Giorgio Agamben, son palpables, pero no podríamos permitirnos profundizar para el resultado de las presentes carillas.

22 "D'ailleurs, bientôt l'art moderne aura à subir un autre examen encore plus sévère et où se refléteront les partialités nées des conditions nouvelles: le futur s'approche, il aura évolué, il éprouvera d'autres lassitudes, d'autres désirs, et il reprochera à l'art d'aujourd'hui d'être solidaire de ce passé, qu'il a pourtant cru détruire" (T. del A.). 
Lo que vemos, es que en Huyghe, la crítica se prolonga a los continuadores de los "estilos" o "vanguardias" europeos, y que si bien dicen responder a estas, lo que hacen es replicar procedimientos de obra.

Por lo tanto, volviendo a Agamben, en las zonas oscuras del arco de la historia, la obra contemporánea sería aquella sin explicación o elocuencia, llena de sustancia, referencias, procedimientos actuales y antiguos, y sin embargo imposible de retorizar para el artista. Puesto la obra contemporánea, para el mismo artista, es absolutamente desconocida.

El Instituto de Extensión de Artes Plásticas que había dirigido Marco Bontá, produce en el Museo Nacional de Bellas Artes en Santiago, para 1950, la exposición De Manet hasta nuestros días. Esta muestra llevó a más de treinta mil visitantes, lo cual para los albores de esa década significó un récord a todas luces. En esta muestra $\mathrm{Hu}$ yghe participó como comisario, siendo una de las muchas colaboraciones que haría para Chile. Él mismo comentando dicha exposición señaló refiriéndose a quienes fueron asistentes "(...) a los vagidos de una génesis todavía incierta o a la disolución última de la creación” (Bindis, 1960, 171). Dando cuenta de esta manera que el arte moderno, iniciado por Manet, podría ser sin lugar a dudas, sujeto de críticas por su falta de actualidad. Ahora, la exposición se caracterizó porque no hubo máximas de superación o comparación. No hay una lectura formalista de la producción artística, en tanto superación de los estilos.

La generación de becarios que iniciaron sus viajes para 1928, alcanzaron a conocer en aquel momento de entreguerras, una producción artística inmensamente estimulante, particularmente en París. Ellos posteriormente regresarían a Chile, cumplen con su periplo venezolano, fundan una institución de extensión nacional e internacional al alero de la Universidad de Chile, siguen a París nuevamente, para apoyar la renovación cultural de la Francia Libre, y finalmente, abren un Museo de Arte Contemporáneo. Ricardo Bindis, sobre De Manet hasta nuestros dias insiste a su vez, que los países que se encuentran en la construcción de un ser nacional, es decir, una identidad local, deben enfrentarse a las condiciones propias de su tiempo. A esto habría que sumar, que las estructuras que presentan obras innovadoras, interpelan tanto al artista como a sus espectadores, y podrían generar estructuras complejas (que retuercen el devenir histórico para hacer carne lo propio). De esto se desprende la posibilidad de una museología contemporánea y de guiones de colecciones donde quien mira reescribe la historia.

\section{Referencias}

Agamben, Giorgio (2012). ¿Qué es lo contemporáneo? Trabajo presentado en el "Curso de Filosofía Teorética”, Facultad de Artes y Diseño de Venecia, Italia. https://etsamdoctorado.files.wordpress.com/2012/12/agamben-que-es-lo-contemporaneo.pdf Allende, Matías (2017). Alcanzar al tiempo y sobrepujar a la tradición. En VV.AA, 
Catálogo Razonado. Colección del MAC (pp. 18-27). Santiago de Chile: Museo de Arte Contemporáneo, Facultad de Artes, Universidad de Chile.

Allende, Matías (2018). De Santiago a Caracas, de Caracas a Santiago. Primer intento de plantear una hipótesis sobre el concepto de cultura contemporánea en América Latina; o lo mismo que definir el color de las escamas de un salmón o encontrar a María Lionza. Anuario. Grupo de investigación Archivo MAC. 92- 107. http://www. mac.uchile.cl/content/documento/2018/enero/anuariogmac_web2.pdf

Barreiro-López, Paula (2015). Del ojo al espectador. Visualidad, crítica y nueva historia del arte. Revista Boletín de Arte. Na15, 18-27. http://papelcosido.fba.unlp. edu.ar/ojs/index.php/boa/article/view/14

Berríos, Pablo y Eva Cancino (2018). Un tiempo sin fisuras. La institución moderna del arte en Chile (1947-1968) Santiago de Chile: Estudios de Arte.

Bindis, R. (1960). La renovación pictórica chilena en el decenio 1950-1960. Anales de la Universidad de Chile (120). 171-174. doi:10.5354/0717-8883.2010.27388

Gagnon, C. (juin - été 1987). Interview avec René Huyghe. Vie des arts, vol. 32 (num. 127), pp: 57-75.

Géraldine, Masson. (juin 2011). Les réseaux professionnels du conservateur de musée : d'une sociabilité informelle à une organisation institutionnelle (1870 - 1940). Trabajo presentado en el Séminaire doctoral commun d'histoire de l'art et d'archéologie Paris 1/Paris 4 - 2010/2011, París, Francia. https://hicsa.univ-paris1.fr/documents/pdf/Ecole\%20doctorale/Geraldine\%20Masson.pdf

Huyghe, René. (1949). Les Contemporains. Paris: Tisné.

Huyghe, René (Dir.). (1935). Histoire de l'art contemporain. La Peinture. París: Félix Alcan, 1935.

Ogaz, Damaso. (septiembre de 1957). Museo de Arte Contemporáneo celebró 10 años, Revista de Arte, No 9. Santiago de Chile. pp: 16-21.

Rouquié, Alain. (2016). Une maison pour l'Amérique Latine. L'Amérique Latine de $A$ à $Z$. Revue Le Monde.París: Le Monde.

Suárez San Pablo, Fernando. (2015). La politique de restauration des peintures des musées nationaux (1930-1950). Les Cahiers de l'École du Louvre, No 7. http:// journals.openedition.org/cel/283; DOI: 10.4000/cel.283

VV.AA. (1945). Permanencia da França. Manifestação francesa. Río de Janeiro: Estados Unidos do Brasil.

VV.AA. (1946). Ars Americana est une exposition de peintures, art populaire et photos du Chili, l'Argentine et l'Uruguay. Paris: Maison de l'Amérique Latine. 